\title{
Involvement of small intestinal motility in blood glucose response to dietary fibre in man
}

\author{
BY CHRISTINE CHERBUT ${ }^{1 *}$, S. BRULEY DES VARANNES', M. SCHNEE ${ }^{2}$, \\ MARTINE RIVAL ${ }^{1}$, J-P. GALMICHE ${ }^{2}$ AND J. DELORT-LAVAL ${ }^{1}$ \\ ${ }^{1}$ Institut National de la Recherche Agronomique, BP 527, 44026 Nantes Cedex 03, France \\ ${ }^{2}$ Equipe Fonctions Digestives et Nutrition, Hôpital $G$ et $R$ Laënnec, Nantes, France
}

(Received 5 April 1993 - Revised 22 July 1993 -Accepted 2 September 1993)

\begin{abstract}
Three dietary fibres with different physicochemical properties were studied in healthy humans for their effects on small intestinal motility and postprandial hyperglycaemia. Duodeno-jejunal motor activity was evaluated electromyographically for $\mathbf{1 8 0}$ min in six subjects who had ingested a test meal composed of glucose alone or glucose with $15 \mathrm{~g}$ of wheat bran (WB), sugar beet (SB) or ispaghula (I) fibres. Glucose and insulin concentrations were determined during the same period. Each subject received each of the four test meals randomly during a $4 \mathrm{~d}$ period. Addition of SB or I to the glucose meal altered duodeno-jejunal motility. Both of these fibres inhibited stationary contractile activity and increased the propagation length and velocity of propagated activity, whereas addition of WB had no effect. These results could reflect the high water-holding capacity of SB and I. Blood glycaemic response to the glucose meal was reduced by SB and I but remained unchanged with WB. Postprandial blood glucose levels were significantly correlated with the total motility index $(r \quad 0.82)$ and stationary activity $(r 0.79)$. Taken together, these observations suggest that the contractile activity induced by dietary fibre in the small intestine probably plays a major role in delayed glucose absorption.
\end{abstract}

Dietary fibre: Glycaemia: Electromyography: Intestinal motility: Man

Dietary fibre (DF) regulates digestive transit time (Cummings, 1978; Read, 1986; Cherbut et al. 1991), mainly through distension of colonic walls by water-rich bulky residues (Eastwood \& Morris, 1992), and also has an effect on small-intestinal motility. In dogs, addition of DF to meals affected postprandial jejunal motility (Bueno et al. 1981; Russell \& Bass, 1986). Moreover, the physical form of DF appears to determine intestinal contractile patterns. Dietary fibres forming discrete particles, such as bran or $\alpha$-cellulose, elicit propagated contraction clusters, whereas gel-forming fibres, such as guar gum, induce disorganized contractions. In pigs, the frequency and energy of the latter contractile pattern depend on chymous viscosity (Cherbut et al. 1990).

There are no studies in the literature concerning DF effects on human small-intestinal motility. However, it is reasonable to suppose that such effects occur and act on digestion and absorption. These effects may be involved in the decreased postprandial hyperglycaemia elicited by some fibres (Schwartz \& Levine, 1980; Torsdottir et al. 1989; Braaten et al. 1991). Lowering of plasma glucose levels has been related to delayed mouthto-caecum transit time (Jenkins et al. 1978) and absorption impairment (Edwards \& Read, 1990). Both mechanisms may result from a change in small-intestinal motility since upper gut contractile activity regulates food transit through the small intestine as well as the degree to which food is mixed with digestive enzymes and the exposure of digestive nutrients to the absorbing surface.

\footnotetext{
* For reprints.
} 
The purpose of the present study was to evaluate the effects of three DF with different physicochemical properties on human small-intestinal motility. Subjects were fed with one of the three DF in a glucose solution. The duodeno-jejunal motor pattern was then studied by analysing electrical activity recorded along $800 \mathrm{~mm}$ of upper small intestine (Bruley des Varannes et al. 1992), with measurement of blood glucose and insulin concentrations at the same time. The results suggest that alteration of postprandial myoelectrical activity in the small intestine is involved in reduction of hyperglycaemia induced by DF with a high waterholding capacity.

\section{METHODS \\ Subjects}

Six healthy male volunteers, 21 to 32 years of age, participated in the study. Informed consent was obtained from each subject, and the study was approved by the Ethics Committee of the Nantes University Hospital. All subjects were carefully interviewed before entry into the study. None had a prior history of gastrointestinal disease or food allergies or used medications during the study.

\section{Meals}

The four experimental meals studied were composed of $125 \mathrm{ml}$ of a glucose solution $(200 \mathrm{~g} / \mathrm{l})$ alone $(\mathrm{G})$, and $\mathrm{G}$ with added $15 \mathrm{~g}$ of wheat bran (WB), sugar beet (SB) or ispaghula husk (I) fibres. Wheat-bran and sugar-beet fibres were processed by SOFALIA (Paris, France). Ispaghula fibre came from Ispaghul Trouette-Perret ${ }^{\circledR}$ (ETRIS S.A.R.L., Paris, France).

The DF were chemically analysed using the Association of Official Analytical Chemists' method described by Prosky et al. (1988). Their water-holding capacity was determined by centrifugation (Eastwood et al. 1983). DF samples were soaked at $37^{\circ}$ in saline $(9 \mathrm{~g} \mathrm{NaCl} / \mathrm{l})$ with stirring for $24 \mathrm{~h}$. Weighed samples of the soaked DF were then transferred to centrifuge tubes containing saline and centrifuged at $6000 \mathrm{~g}$ for $15 \mathrm{~min}$. The supernatant fraction was discarded and the tubes were left to drain for $30 \mathrm{~min}$ at room temperature. The weight of the wet material in each tube was determined before freeze-drying to estimate the water-holding capacity. The median size of fibre particles was measured by screening a DF sample (100 g) for 15 min using a Buhler MLU 300 laboratory grading sifter (Association Françoise de Normalisation, 1985). The relative viscosity of the test meals was assessed by measuring the flow rate in a viscometer (Visco-F; Kinematica, Kriens-Luzern, Switzerland).

\section{Glucose dialysis retardation index}

The glucose dialysis retardation index of the three DF was determined in vitro, as described by Adiotomre et al. (1990). Dialysis bags (100 mm long; NW 12000 to 14000; Polylabo, Nantes, France) were soaked in a solution of sodium azide $(1 \mathrm{~g} / 1)$. Each bag was filled with $6 \mathrm{ml}$ sodium azide solution and $36 \mathrm{mg}$ glucose alone (control) or with $22 \mathrm{mg}$ of one of the DF. The amounts of DF and glucose were chosen to model the DF: glucose ratio of the experimental meals, i.e. 0.6 g DF/g glucose. Each DF was hydrated in sodium azide solution for $14 \mathrm{~h}$ before dialysis. Each bag was tied, suspended in $100 \mathrm{ml}$ sodium azide solution and placed in a stirred bath at $37^{\circ}$ for $60 \mathrm{~min}$. At 30 and $60 \mathrm{~min}, 2 \mathrm{ml}$ dialysate was analysed for glucose by the glucose oxidase EC method. The glucose dialysis retardation index was calculated as:

$$
100-\frac{\text { (total glucose diffused from the bag containing DF } \times 100 \text { ) }}{\text { (total glucose diffused from the bag with no DF present) }}
$$




\section{Myoelectrical activity recording}

Small-intestinal myoelectrical activity was recorded using a probe made of polyvinyl tubing (length $0.25 \mathrm{~m}$, external diameter $4.0 \mathrm{~mm}$, internal diameter $2.8 \mathrm{~mm}$; Tygon ${ }^{\circledR}$; Prolabo, Paris, France) fitted with eight pairs of nickel-chrome electrodes (diameter $0.12 \mathrm{~mm}$; Trimel $^{\mathbb{R}}$; Microfil Industrie SA, Renens, Switzerland). Inter-electrode distance was $5 \mathrm{~mm}$, and the distance between each pair was $100 \mathrm{~mm}$, allowing myoelectrical activity to be recorded along $800 \mathrm{~mm}$ of small intestine. The probe was designed from the model described by Fioramonti et al. (1980). Validation data for the signal recorded from the probe intraluminal electrodes were previously reported (Kolrep-Dechauffour et al. 1989).

After a $10 \mathrm{~h}$ fasting period and light nasal local anaesthesia (Xylocaine ${ }^{\circledR} \%$; Laboratoire Roger Bellon, Neuilly sur Seine, France), the volunteer was intubated pernasally into the stomach with the probe. After the probe had passed through the pylorus, a latex balloon mounted at the tip was inflated with $10-15 \mathrm{ml}$ air. When the proximal pair of electrodes was $200 \mathrm{~mm}$ beyond the pylorus the balloon was deflated and the probe attached to the nose. Electrical activity was then recorded simultaneously from the eight pairs of electrodes on an eight-channel EEG polygraph (mini-VIII; Alvar Electronique, Paris, France) set at a time constant of $0.01 \mathrm{~s}$ and a paper speed of $2.5 \mathrm{~mm} / \mathrm{s}$. At the same time, total activity was calculated every minute by integrator circuits and automatically plotted on a microcomputer (Latour \& Ferré, 1985).

\section{Analysis of data}

Recordings of electrical activity were analysed visually by two of the authors (CC and SBV). Several activity patterns were distinguished, as previously described (Bruley des Varannes et al. 1992).

Phase 3 of the migrating motor complex (MMC) was defined by the regular occurrence of spike bursts at a frequency of 10 to 12 cycles $/ \mathrm{min}$. These phases were characterized by their duration and propagation velocity.

Postprandial activity (PPA), defined by the myoelectrical activity recorded between the meal ingestion and the first following phase 3 , consisted of alternating periods of irregular activity and quiescence. In PPA, two types of electrical activity patterns were analysed, rushes and minute-rhythms, characterized by frequency, duration, propagation length and velocity. Rushes (Fig. 1) consisted of high-amplitude electrical activities ( $>200 \mu \mathrm{V}$ ) lasting for $9 \cdot 3(\mathrm{SE} 0 \cdot 3) \mathrm{s}$, propagating on at least three successive sites $(>300 \mathrm{~mm})$. Minuterhythms (Fig. 1) consisted of low-amplitude electrical activities $(<100 \mathrm{mV})$, appearing sequentially and characterized by a duration of 40.4 (SE 1.2$) \mathrm{s}$, propagating on at least three sites, and separated by intervals of electrical inactivity lasting $54 \cdot 7$ (SE 2.5) s.

At every minute, the microcomputer automatically determined a relative motility index (MI) by dividing the quantity of postprandial electrical activity (calculated as (spike burst maximal power $\times$ duration) $\times 0.5$ (Lucas et al. 1991)), by the mean quantity of electrical activity/min recorded during preprandial phase 3 which represented theoretical maximal myoelectrical activity.

\section{Experimental design}

Each volunteer was studied on four occasions separated by $7 \mathrm{~d}$. Small-intestinal activity was recorded first in the fasting period until occurrence of phase 3 of the migrating motor complex (MMC). Five min after the end of phase 3 on the distal recorded level, one of the four experimental meals, according to a randomized predetermined order, was administered to the volunteer in $2 \mathrm{~min}$. The recording was then continued for at least $3 \mathrm{~h}$.

Blood samples for glucose and insulin analysis were taken $15 \mathrm{~min}$ before and immediately before the meal was ingested and then at $15 \mathrm{~min}$ intervals thereafter for $3 \mathrm{~h}$. Plasma glucose 
(a)

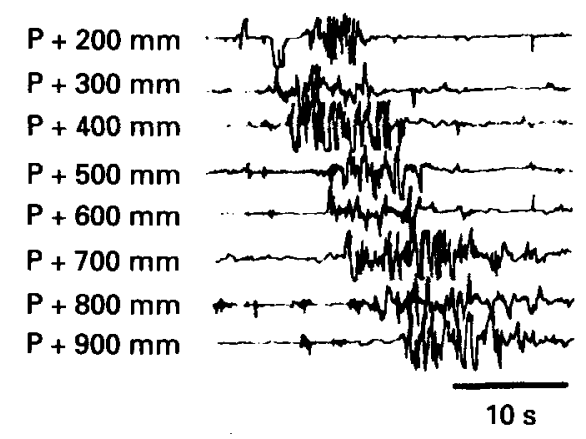

(b)

$$
\begin{aligned}
& P+200 \mathrm{~mm} \\
& P+300 \mathrm{~mm} \\
& P+400 \mathrm{~mm} \\
& P+500 \mathrm{~mm} \\
& P+600 \mathrm{~mm} \\
& P+700 \mathrm{~mm} \\
& P+800 \mathrm{~mm} \\
& P+900 \mathrm{~mm}
\end{aligned}
$$

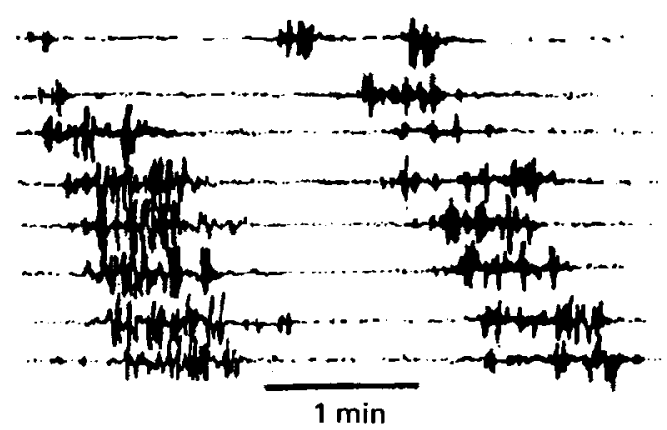

Fig. 1. Propagated myoelectrical activities, one rush $(a)$ and two minute-rhythms $(b)$, observed in the postprandial period in a healthy volunteer. The eight contact electrodes are spaced $100 \mathrm{~mm}$ apart and located in the proximal small intestine. $P$, pylorus.

was measured using the glucose oxidase method (Beckman Analyzer, Fullerton, CA, USA), and plasma insulin was determined by radioimmunoassay (Oris, Gif sur Yvette, France).

\section{Statistical analysis}

Results are expressed as means with their standard errors (SE).

Fibre-glucose-dialysis retardation indexes were compared with controls by a Mann-Whitney $U$ test. The effect of subjects on myoelectrical activity data and plasma glucose and insulin values was tested by one-way analysis of variance (ANOVA). As there was no significant effect of subjects on the tested variables, except on the number of rushes and minute-rhythms, another one-way ANOVA was performed to test the effect of test meals on these variables. When there was a statistically significant effect of test meals, means were compared using the Fisher $F$ test of probable least significant difference. Linear correlations between individual motility index and plasma results were calculated using the least square method. Each point of the regressions represents the data from one subject on one diet. The regressions are therefore based on a mix of between and within subject variation. Statistical analysis was performed using StatViews 512 (version 1.01) software (Brain Power Inc., Calabas, CA, USA).

RESULTS

Dietary fibre characteristics

Chemical and physicochemical characteristics of the DF are given in Table 1 . The flow rates of the DF test meals were comparable, 25.4, 20.1 and $16.1 \mathrm{~mm} / \mathrm{s}$ (means for two 
Table 1. Physical and chemical characteristics of dietary fibres

(Values are means for two determinations per fibre source)

\begin{tabular}{lcccc}
\hline & $\begin{array}{c}\text { Total fibre content } \\
(\mathrm{g} / \mathrm{kg} \text { fibre source) }\end{array}$ & $\begin{array}{c}\text { Soluble fibre content } \\
\text { (g/kg fibre) }\end{array}$ & $\begin{array}{c}\text { Water-holding } \\
\text { capacity } \\
\text { (g/g fibre) }\end{array}$ & $\begin{array}{c}\text { Particle size } \\
\text { (mm) }\end{array}$ \\
\hline Wheat bran & 586 & 169 & 5 & $0 \cdot 64$ \\
Sugar beet & 875 & 235 & 18 & $0 \cdot 57$ \\
Ispaghula husk & 915 & 92 & 12 & $0 \cdot 68$ \\
\hline
\end{tabular}

Table 2. Effect of dietary fibres on glucose concentration ( $\mathrm{mmol} / \mathrm{l}$ ) in dialysate in vitro* (Values are means with their standard errors for three determinations per diet)

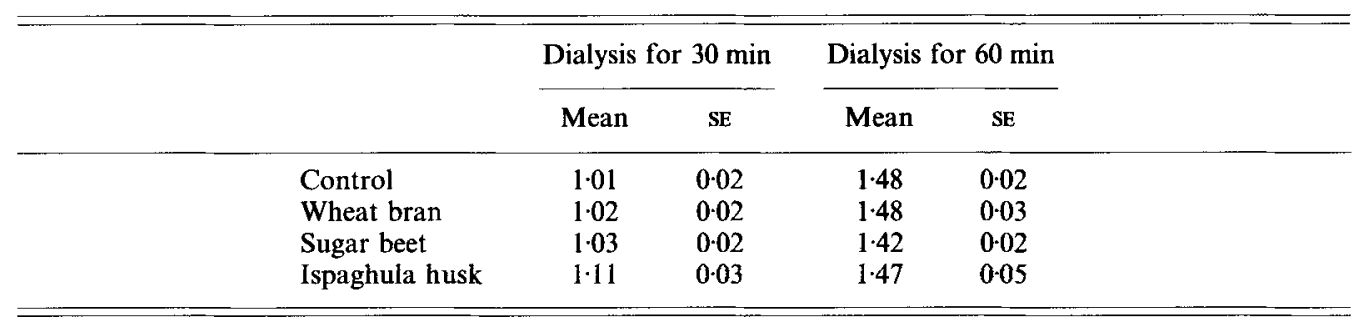

* For procedure, see p. 676.

determinations) for WB, SB and I respectively, and slightly lower than that of $\mathrm{G}$ ( > $30 \mathrm{~mm} / \mathrm{s}$ ). The DF had no significant retarding effect on glucose movement in the dialysis system in vitro (Table 2), and the glucose dialysis retardation indexes were not different from zero.

\section{Phase 3}

Regardless of the meal, the mean duration of phase 3 (6.9 (SE 0.2) min), was not different between fasting and postprandial periods. However, the propagation velocity was higher in the fasting period (69 (SE 3) $\mathrm{mm} / \mathrm{min})$ than after the meal $(51$ (SE 2$) \mathrm{mm} / \mathrm{min}(P=$ $0.0001)$ ), but was not changed by the DF $(P=0.23)$.

Occurrence of the first phase 3 after the meal was not significantly different after $G$ and the other meals (G 99.9 (SE 15.4) min; WB 87.9 (SE 16.9) min; SB 65.6 (SE 11.5) min; I $83 \cdot 1$ (SE 15.5) $\mathrm{min}, P=0 \cdot 51$ ).

\section{Postprandial activity}

During the postprandial period, the number of rushes and minute-rhythms differed between volunteers $(P=0.0001)$. Furthermore, a highly significant inverse correlation was found between the number of rushes and minute-rhythms $(y=0.51 x+23 ; r 0.86, n 22, P=$ 0.0001). However, the mean hourly frequency of rushes and minute-rhythms (3.9 (SE 0.1) and 5.9 (SE 0.6) respectively) was not different between the four meals. SB and I induced longer and faster propagation for both rushes and minute-rhythms than did G and WB $(P<0.05$; Table 3).

Except for rushes and minute-rhythms, the PPA was composed of spike bursts which did not appear to be organized nor propagated over more than $100 \mathrm{~mm}$. This postprandial 
Table 3. Characteristics of propagation of postprandial rushes and minute-rhythms in the human small intestine after ingestion of test meals composed of glucose alone $(G)$ or $G+$ fibres of wheat bran, sugar beet or ispaghula husk*

(Values are means with their standard errors for $n$ determinations)

\begin{tabular}{|c|c|c|c|c|c|c|c|c|c|c|c|c|}
\hline & \multicolumn{6}{|c|}{ Rushes } & \multicolumn{6}{|c|}{ Minute-rhythms } \\
\hline & \multicolumn{3}{|c|}{$\begin{array}{c}\text { Length of } \\
\text { propagation }(\mathrm{mm})\end{array}$} & \multicolumn{3}{|c|}{$\begin{array}{l}\text { Velocity of propagation } \\
\qquad(\mathrm{mm} / \mathrm{min})\end{array}$} & \multicolumn{3}{|c|}{$\begin{array}{c}\text { Length of } \\
\text { propagation }(\mathrm{mm})\end{array}$} & \multicolumn{3}{|c|}{$\begin{array}{l}\text { Velocity of propagation } \\
\qquad(\mathrm{mm} / \mathrm{min})\end{array}$} \\
\hline & Mean & $\mathbf{S E}$ & $n$ & Mean & SE & $n$ & Mean & SE & $n$ & Mean & SE & $n$ \\
\hline Glucose & $572^{\mathrm{a}}$ & 19 & 50 & $17^{\mathrm{a}}$ & 1 & 50 & $621^{\mathrm{a}}$ & 11 & 71 & $15^{\mathrm{a}}$ & 1 & 71 \\
\hline Wheat bran & $554^{a}$ & 24 & 26 & $16^{\mathrm{a}}$ & 2 & 26 & $663^{b}$ & 7 & 113 & $16^{\mathrm{ab}}$ & 1 & 113 \\
\hline Sugar beet & $605^{a b}$ & 20 & 38 & $20^{\mathrm{b}}$ & 1 & 38 & $659^{b}$ & 12 & 44 & $20^{\mathrm{b}}$ & 2 & 44 \\
\hline Ispaghula husk & $633^{b}$ & 20 & 33 & $20^{\mathrm{b}}$ & 1 & 33 & $671^{b}$ & 6 & 110 & $25^{c}$ & 2 & 110 \\
\hline
\end{tabular}

a,b, , Values with unlike superscript letters within a column were significantly different $(P<0-05)$.

* For details of diets and procedures see pp. 676-678.
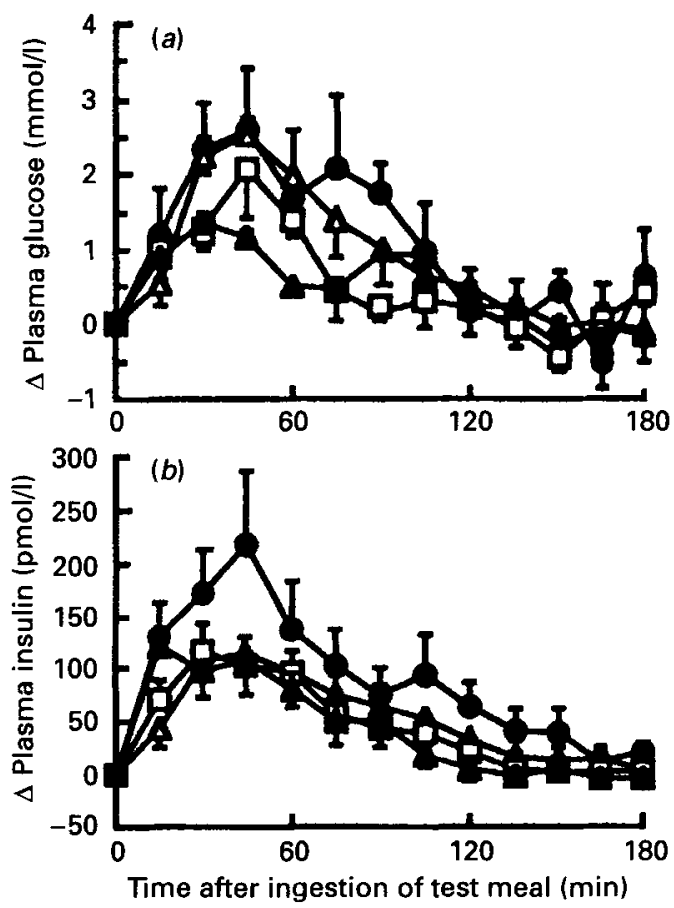

Fig. 2. Incremental plasma glucose $(a)$ and insulin $(b)$ responses (mean and sE as vertical bars) to a test meal composed of $(\Theta)$ glucose $(G)$ alone, or $(\triangle) G+$ wheat bran fibre, $(\square) G+$ sugar-beet fibre or $(\Delta) G+$ ispaghulahusk fibre, in six healthy subjects. For details, see pp. 676-678. 

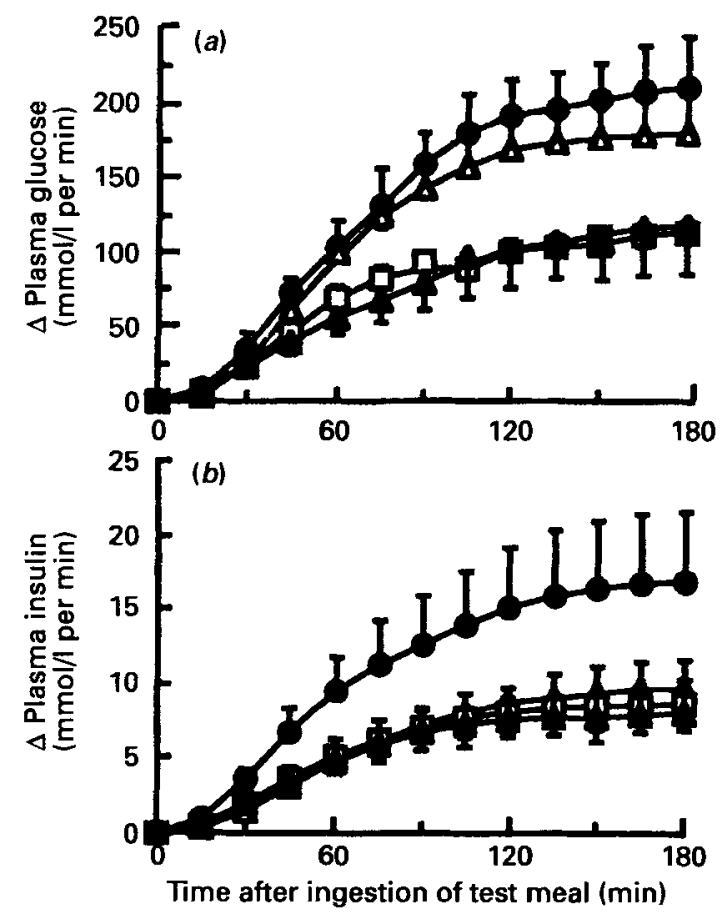

Fig. 3. Cumulative area under the curve (mean and SE as vertical bars) of incremental plasma glucose (a) and insulin $(b)$ after ingestion of test meal composed of $(O)$ glucose $(G)$ alone, or $(\triangle) G+$ wheat bran fibre, $(\square)$ $\mathrm{G}+$ sugar-beet fibre or $(\Delta) \mathrm{G}+$ ispaghula-husk fibre, in six healthy subjects. For details, see pp. 676-678.

stationary activity occurred for 27.8 (SE $2 \cdot 2$ ) $\%$ and 27.5 (SE 3.3 ) $\%$ of the recording time respectively after $G$ and $W B$, but was reduced to 17.0 (SE 5.0) and 11.6 (SE 5.2)\% respectively after $\mathrm{SB}$ and $\mathrm{I}(P=0.03)$.

\section{Motility index}

During the postprandial period, the relative MI was higher following G and WB $(0.87$ (SE 0.15) and 1.00 (SE 0.26)) than after SB and I (0.62 (SE 0.13) and 0.69 (SE 0.14)), although the difference was not statistically significant $(P=0.49)$. However, there was a significant relationship between the relative MI and occurrence of stationary activity $(y=3.97 x+0.08$, $r 0.70, n 24, \mathrm{P}=0.002$ ).

\section{Glycaemia and insulinaemia}

The DF meals caused lower insulin peaks than did glucose alone, while only SB and I decreased the hyperglycaemic response to glucose (Fig. 2). However, these reductions were not statistically significant. Areas under the curve for glucose and insulin (AUCg and AUCi) depended on the type of meal. The AUCg were higher after $G$ and WB than after SB and I ( $P$ ranged between 0.07 and 0.04 from 45 to $180 \mathrm{~min}$ ), but the three DF meals led to the same AUCi which were smaller than the areas measured after $\mathrm{G}(P<0.05$ at 30,45 and 60 min, Fig. 3).

The AUCg measured between meal ingestion and glucose peak time were correlated with the mean MI calculated during the same period $(y=19 \cdot 1+27 \cdot 9 x, r 0 \cdot 82, n 22, P=0.0001)$. 


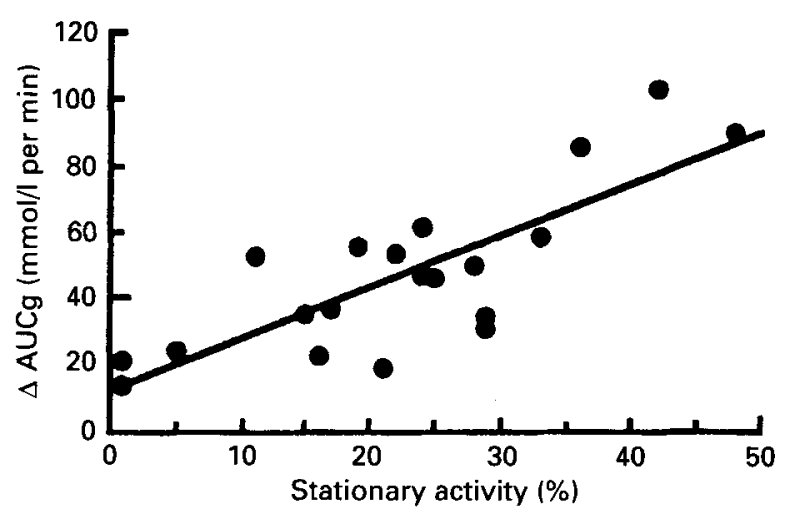

Fig. 4. Correlation between the individual incremental areas under the curve of plasma glucose $(\Delta \mathrm{AUCg})$ and the percentage of stationary motor activity measured between test meal ingestion and plasma glucose peak time in six healthy subjects. $y=12.3+155.8 x, r 0.79, n 22, P=0.0001$.

There was also a significant correlation between these $\mathrm{AUCg}$ and the percentages of stationary activity $(y=12 \cdot 3+155 \cdot 8 x, r 0 \cdot 79, n 22, P=0 \cdot 0001$; Fig. 4).

\section{DISCUSSION}

The present study shows that DF influenced postprandial myoelectrical activity in human small intestine. Addition of sugar-beet and ispaghula-husk DF to a glucose meal reduced the occurrence of stationary activity and simultaneously increased the propagation length and velocity of propagated activity. Conversely, wheat bran DF did not affect smallintestinal activity. In addition, postprandial glycaemic responses appeared to be related to motor effects induced by the meals in the small intestine.

To ensure good detection of DF effects, it was essential to minimize the interference of the recording system with small-intestinal motility. Electromyography was considered preferable to the classic perfused catheters used for manometric assessment because no intraluminal perfusion was involved. The sensitivity and predictive value of the recording system during interdigestive and postprandial periods were previously tested in pigs fitted with both intraparietal electrodes and the intraluminal probe. After the animals were given a $1.5 \mathrm{~kg}$ meal composed of $500 \mathrm{~g}$ dry matter and 11 water, intraluminal electrode sensitivity and predictive value were 86 (SE 3) \% and 90 (SE 3) \% respectively, thus not different from percentages for the interdigestive period (Kolrep-Dechauffour et al. 1989).

Postprandial myoelectrical activity in the small intestine consists of both aborally propagated and stationary randomly-appearing spike bursts (Staumont et al. 1992). Propagated activity itself consists of two different patterns, rushes and minute-rhythms (Bruley des Varannes et al. 1992). In some volunteers in the present study, small-intestinal myoelectrical activity was preferentially organized as rushes and in others mainly as minute-rhythms. This observation, confirmed by the negative correlation between the number of rushes and minute-rhythms, suggests that these two motility patterns may have close functional consequences in terms of movement of luminal contents.

The DF influenced postprandial activity, not by modifying the reappearance of phase 3 after the meal but by changing the patterns of propagated and stationary myoelectrical activity. The stationary activity occurred for about $30 \%$ of recording time after $\mathrm{G}$ and $\mathrm{WB}$, but decreased dramatically to about $15 \%$ after SB and I. The latter two DF also increased 
propagation length and velocity for both rushes and minute-rhythms, whereas WB had no effect.

The changes in postprandial activity could be related to the physicochemical properties of sugar-beet and ispaghula-husk DF. The water-holding capacity of both of these DF is higher than that of wheat bran and may thus have increased the volume of gastrointestinal contents. Luminal volume is one of the numerous factors controlling gastrointestinal motility (Malagelada \& Azpiroz, 1989). Mechanoreceptors within the visceral wall are excited by distension, which reflexly modifies gastric and small-intestinal motility (Grundy \& Scratcherd, 1989; Hölzer \& Raybould, 1992). In dogs, insoluble fibre particles decrease the number of isolated contractions (Bueno et al. 1981) and convert the contractile patterns into aborally propagated, clustered contractions (Russell \& Bass, 1986). In the present study, organized and propagated activity was similarly favoured by sugar beet and ispaghula-husk DF since stationary activity was inhibited.

Glycaemic response to glucose was not changed by wheat bran but was significantly decreased by sugar-beet and ispaghula-husk DF. A similar reduction in postprandial hyperglycaemia has been observed after meals enriched with these two DF (Sud et al. 1988; Morgan et al. 1990). This effect might be related to the water-holding capacity of the fibres, which could trap glucose within their physical structure (Eastwood \& Morris, 1992). However, as the fibres were unable to retard glucose movement in vitro, it is unlikely that the flattening of glycaemic responses was caused by glucose retention in sugar-beet and ispaghula-husk DF matrices.

The increased ratio of propagated to stationary activity might indicate the effect of sugarbeet and ispaghula-husk DF on postprandial hyperglycaemia. Absorption of glucose depends in part on intestinal contractions which ensure mixing of luminal contents and create convective currents to bring nutrients from the bulk phase towards the epithelia (Edwards \& Read, 1990). In vitro studies have demonstrated that absorption of water, electrolytes, and glucose is enhanced by intestinal wall movements (Macagno et al. 1982; Edwards et al. 1988). In canine isolated jejunum, glucose absorption was lowest during quiescent phase 1 of the MMC and then increased during phases 2 and 3, finally becoming maximal after feeding (Sarr et al. 1980). The decrease in stationary activity after SB and I might have reduced the mixing of luminal contents, thus impeding glucose from reaching the absorptive surface. This hypothesis is supported by the significant correlation between glycaemic areas and stationary activity.

SB and I might also have impaired glucose absorption by enhancing aboral propulsion of luminal contents through the small intestine since the propagation length and velocity of propagated activity were increased after their ingestion. According to Siegle \& Ehrlein (1988), the length of contraction and the ratio of propagated to stationary contractions are the main factors influencing the transit of luminal contents. Hence, the lengthening of propagation and the inhibition of the stationary activity induced by sugar-beet and ispaghula-husk DF should have speeded up the transit of luminal contents. It has been reported that insoluble DF can accelerate orocaecal transit in humans (Hanson \& Winterfeldt, 1985; Hamberg et al. 1989), whereas soluble, viscous DF, such as guar gum, delay small-intestinal transit time, mainly through a slowing effect in the ileum (Brown et al. 1988). It is thus possible that sugar-beet and ispaghula-husk insoluble DF reduced transit time in the proximal small intestine, thereby shortening glucose contact time with the absorbing surface.

It may be concluded that DF act on small-intestinal motility and that their effects seem to have been involved in the decrease in postprandial glycaemia. DF with a high waterholding capacity inhibited postprandial stationary activity and simultaneously increased the propagation length and velocity of propagated activity. Both of these motor effects 
could account for the limited access of glucose to the absorbing surface and thus the flattening of glycaemic responses.

We thank Marie-France Courjal and G. Lecannu for their helpful technical assistance.

\section{REFERENCES}

Adiotomre, J., Eastwood, M. A., Edwards, C. A. \& Brydon, W. G. (1990). Dietary fiber: in vitro methods that anticipate nutrition and metabolic activity in humans. American Journal of Clinical Nutrition 52, 128-134.

Association Françoise de Normalisation (1985). Représentation des distributions granulométriques (Granulometric distributions). Norme française $X-11-635,19 \mathrm{p}$.

Braaten, J. T., Wood, P. J., Scott, F. W., Riedel, K. D., Poste, L. M. \& Collins, M. W. (1991). Oat gum lowers glucose and insulin after an oral glucose load. American Journal of Clinical Nutrition 53, 1425-1430.

Brown, N. J., Worlding, J., Rumsey, R. D. E. \& Read, N. W. (1988). The effect of guar gum on the distribution of a radiolabelled meal in the gastrointestinal tract of the rat. British Journal of Nutrition 59, 223-231.

Bruley des Varannes, S., Cherbut, C., Schnee, M., Delort-Laval, J. \& Galmiche, J. P. (1992). Effects of lactulose on fasting small intestine myoelectrical activity in humans. European Journal of Gastroenterology and Hepatology 4, 539-545.

Bueno, L., Praddaude, F., Fioramonti, J. \& Ruckebusch, Y. (1981). Effect of dietary fiber on gastrointestinal motility and jejunal transit time in dogs. Gastroenterology 80, 701-707.

Cherbut, C., Albina, E., Champ, M., Doublier, J. L. \& Lecannu, G. (1990). Action of guar gums on the viscosity of digestive contents and on the gastrointestinal motor function in pigs. Digestion 46, 205-213.

Cherbut, C., Salvador, V., Barry, J. L. \& Delort-Laval, J. (1991). Dietary fibre effects on intestinal transit in man: involvement of their physicochemical and fermentative properties. Food Hydrocolloids 5, 15-22.

Cummings, J. H. (1978). Diet and transit through the gut. Journal of Plant Foods 3, 83-95.

Eastwood, M. A. \& Morris, E. R. (1992). Physical properties of dietary fiber that influence physiological function: a model for polymers along the gastrointestinal tract. American Journal of Clinical Nutrition 55, 436-442.

Eastwood, M. A., Robertson, J. A., Brydon, W. G. \& McDonald, D. (1983). Measurement of water-holding properties of fibre and their faecal bulking ability in man. British Journal of Nutrition 50, 539-547.

Edwards, C. A. \& Read, N. W. (1990). Fibre and small intestinal function. In Dietary Fibre Perspectives, vol. II, pp. 52-75 [A. R. Leeds, editor]. London: John Libbey.

Edwards, C. A., Johnson, I. T. \& Read, N. W. (1988). Do viscous polysaccharides slow absorption by inhibiting diffusion or convection? European Journal of Clinical Nutrition 42, 307-312.

Fioramonti, J., Bueno, L. \& Frexinos, J. (1980). Sonde endoluminale pour l'exploration électromyographique de la motricité colique chez l'homme (An endoluminal probe for electromyographic exploration of colonic motility in man). Gastroenterologie Clinique et Biologique 4, 546-550.

Grundy, D. \& Scratcherd, T. (1989). Sensory afferents from the gastrointestinal tract. In Handbook of Physiology "The Gastrointestinal System", vol. 6, part 1, pp. 593620 [J. D. Wood, volume editor]. Bethesda: American Physiological Society.

Hamberg, H., Rumessen, J. J. \& Gudmand-Hoyer, E. (1989). Blood glucose response to pea fiber: comparison with sugar beet and wheat bran. American Journal of Clinical Nutrition 50, 324-328.

Hanson, C. F. \& Winterfeldt, E. A. (1985). Dietary fiber effects on passage rate and breath hydrogen. American Journal of Clinical Nutrition 42, 44-48.

Hōlzer, H. H. \& Raybould, H. E. (1992). Vagal and splanchnic sensory pathways mediate inhibition of gastric motility induced by duodenal distension. American Journal of Physiology 262, G603-G608.

Jenkins, D. J. A., Wolever, T. M. S., Leeds, A. R., Gassull, M. A., Haisman, P., Dilawari, J., Goff, D. V., Metz, G. L. \& Alberti, K. G. M. M. (1978). Dietary fibres, fibre analogues, and glucose tolerance: importance of viscosity. British Medical Journal 1, 1392-1394.

Kolrep-Dechauffour, S., Cherbut, C., Bruley des Varannes, S., Guiheneuc, P. \& Galmiche, J. P. (1989). Les électrodes annulaires endoluminales sont-elles fiables pour le recueil de l'activité myoélectrique de l'intestin grêle (Are annular endoluminal electrodes reliable for recording myoelectrical activity in the small intestine)? Gastroenterologie Clinique et Biologique 13, 602-606.

Latour, A. \& Ferre, J. P. (1985). Computer-aided analysis of gastrointestinal myoelectric activity. Journal of Biomedical Engineering 7, 127-131.

Lucas, M-F., Cherbut, C. \& Bruley des Varannes, S. (1991). Automatic recognition of intestinal myoelectrical activity. Annual International Conference of the IEEE Engineering in Medicine and Biology Society 13, 483-484.

Macagno, E. O., Christensen, J. \& Lee, C. L. (1982). Modeling the effect of wall movement on absorption in the intestine. American Journal of Physiology 243, G541-G550.

Malagelada, J. R. \& Azpiroz, F. (1989). Determinants of gastric emptying and transit in the small intestine. In Handbook of Physiology “The Gastrointestinal System”, vol. 6, part 2, pp. 909-938 [J. D. Wood, volume editor]. Bethesda: American Physiological Society.

Morgan, L. M., Tredger, J. A., Wright, J. \& Marks, V. (1990). The effect of soluble- and insoluble-fibre 
supplementation on post-prandial glucose tolerance, insulin and gastric inhibitory polypeptide secretion in healthy subjects. British Journal of Nutrition 64, 103-110.

Prosky, L., Asp, N. G., Schweizer, T. F., De Vries, J. W. \& Furda, I. (1988). Determination of insoluble, soluble, and total dietary fiber in foods and food products: Interlaboratory study. Journal of the Association of Official Analytical Chemists 71, 1017-1023.

Read, N. W. (1986). Dietary fiber and bowel transit. In Dietary Fiber: Basic and Clinical Aspects, pp. 81-100 [G. Vahouny and D. Kritchevsky, editors). New York: Plenum Press.

Russell, J. \& Bass, P. (1986). Effects of laxative and nonlaxative hydrophilic polymers on canine small intestinal motor activity. Digestive Diseases and Sciences 31, 281-288.

Sarr, M. G., Kelly, K. A. \& Phillips, S. F. (1980). Canine jejunal absorption and transit during interdigestive and digestive motor states. American Journal of Physiology 239, G167-G172.

Schwartz, S. E. \& Levine, G. D. (1980). Effects of dietary fiber on intestinal glucose absorption and glucose tolerance in rats. Gastroenterology 79, 833-836.

Siegle, M. L. \& Ehrlein, H. J. (1988). Digestive motor patterns and transit of luminal contents in canine ileum. American Journal of Physiology 254, G552 G559.

Staumont, G., Delvaux, M., Fioramonti, J., Berry, P., Bueno, L. \& Frexinos, J. (1992). Differences between jejunal myoelectric activity after a meal and during phase 2 of migrating motor complexes in healthy humans. Digestive Diseases and Sciences 37, 1554-1561.

Sud, S., Siddhu, A. \& Bijlani, R. L. (1988). Effect of ispaghula husk on postprandial glycemia and insulinemia following glucose and starch drinks. Nutrition 4, 1-3.

Torsdottir, I., Alpsten, M., Andersson, H. \& Einarsson, S. (1989). Dietary guar gum effects on postprandial blood glucose, insulin and hydroxyproline in humans. Journal of Nutrition 119, 1925-1931. 